\title{
Modelling oil and gas stock returns using multi factor asset pricing model including oil price exposure
}

\begin{abstract}
Oil and gas is one of the most important sectors in every economy and the valuation of oil and gas companies becomes quite challenging due to the volatility of crude oil price. The paper investigates the determinants of the UK oil and gas stock returns using multi factor asset pricing model and the existence of asymmetric effects in the Brent crude oil price. Our results show that market risk, oil price risk, size and book-to-market related factors are all relevant in the determination of asset returns of the oil and gas companies quoted on the London stock exchange. Oil price increases and decreases decomposed separately have more effect on the oil companies' stock returns than the normal log changes of the price which shows the presence of asymmetric effect. However, the oil price shocks in general do not seem to strongly affect stock returns in oil and gas sector possibly due to horizontal and vertical integration of bigger companies in the sector.
\end{abstract}

Keywords: asset pricing models, Brent crude oil, asymmetry in oil price, size effect, book to market ratio, oil and gas sector, oil price exposure, structural breaks.

JEL Classification Codes: G12 


\section{Introduction}

One of the biggest challenges in the field of finance is how to effectively model the risk and return of financial securities. Researchers have formulated various asset pricing models that tend to explain the determinants of asset returns. Markowitz's (1952) mean-variance analysis provides the foundations of portfolio optimisation. Sharpe (1964) and Lintner (1965) developed a single factor model commonly known as the Capital Asset Pricing Model (CAPM). The main assumption in this model is that asset return is determined by an asset's systematic risk since unsystematic risks of individual assets can be eliminated by diversification in an efficient portfolio. The main criticism of the CAPM is its failure to consider size, value and momentum effect in asset returns. These anomalies have resulted in modifications to the single factor model. Multi-factor asset pricing models such as that of Fama and French's (1993) three factor model and Fama, French and Carhart's (1997) four-factor asset pricing models have been developed to consider other relevant factors in the determination of asset returns. In recent years, the impact of underlying commodity prices such as oil price and its shocks have been incorporated into multi-factor asset pricing models to analyse stock price dynamics. Broadstock et al (2016) had made an assessment of oil shocks and stock returns of Chinese firms using Fama-French factors. For other similar studies see also Narayan and Sharma (2011), Phan et al (2015a) and Melichar (2016).

In this study we aim to investigate the determinants of asset pricing in the UK oil and gas stocks quoted on the London stock exchange. We plan to adopt a multifactor asset pricing model like Fama-French-Carhart (1997) model augmented with oil price represented by the Brent crude oil price. The asymmetry in the oil price will also be tested in the same type of model to find the influence of oil price 
increases as well as decreases. Structural breaks were incorporated into the models to account for the global financial crisis of 2007 to 2008 and the recent falling oil prices between 2014 and 2015.

\section{Review of Literature}

The initial proposition of the Capital Asset Pricing Model (CAPM) was derived from the works of Sharpe (1964) and Lintner (1965) as an extension of Markowitz's mean-variance analysis model (also supported by Mossin (1966)). Fama and French (1993) proposed a three factor asset pricing model designed to overcome some of the limitations observed in CAPM by introducing additional factors like firm size and book to market ratio along with the market risk. Since then, scholars have been confirming the power of the new model in the determination of stock returns than the original CAPM, (Lawrence et al (2007); Gregory et al (2013)). Carhart (1997) suggested the importance of a firm's recent performance in its ability to outperform in near future and introduced the fourth factor of momentum effect. The models have been subject to rigorous testing over the years and multi-factor models seem to have slightly more explanatory power the simple CAPM.

The multi-factor asset pricing models have also been used by researchers to assess the impact of commodity prices on stock returns of firms presumed to have association with that commodity. Oil price risk exposure is one of the most tested in the literature because of the pervasive nature of oil prices. Faff and Brailsford (1999) investigated the impact of oil price on the Australian stock market using a two-factor model including beta and oil price as risk factors. The oil price risk factor's was found significant in the oil and gas, paper, packaging and transport sector. Other firms seemed to be able to transfer most of the oil price risk to 
customers or managed it with hedging. Phan et al (2015b) investigated the effect of oil price changes on stock returns of oil producers and oil consumers using a similar methodology adopted by Narayan and Sharma (2011) and Arouri (2011). Stock returns of oil producers were found to be positively affected by oil price changes regardless of whether it was an oil price increase or decrease. Asymmetric effects in oil shocks were tested by scholars such as Mork et al (1994) and Mendoza and Vera (2010). Mork et al (1994) studied the macroeconomic responses to the asymmetry in oil prices in some OECD countries and discovered the presence of asymmetric effects in the correlation between oil price and GDP fluctuations. Oil price increases were found to be negatively correlated with GDP fluctuations in most of the countries. Mendoza and Vera (2010) found that oil price increases has more effect on the Venezuelan economy than oil price decreases.

The relationship between oil shocks and oil and gas stock returns of Central and Eastern Europe (CEE) markets (Czech Republic, Hungary, Poland, Romania, Slovenia, and Austria) was examined by Mohanty et al (2010) using a two-factor model similar to the one used by Faff and Brailsford (1999). Contrary to the findings of Faff and Brailsford (1999), Mohanty et al (2010) found no significant relationship between oil prices and stock returns over the period of the study between 1998 and 2010. Mohanty and Nandha (2011) estimated the oil price risk exposure of the US oil and gas sector using Fama-French-Carhart's four factor asset pricing model. The model was expanded by an additional risk factor of the monthly changes in oil price using West Texas Intermediate (WTI). The results of this study show that systematic risk, size, book-to-market ratio and fluctuation in oil price are all significant and explain variations in the oil and gas stock returns in the USA. The impact of oil price fluctuation varies over time and also depends on firm type and industry subsectors (exploration, equipment services and integrated 
oil and gas). The risk exposure was found to be higher in exploration and oil equipment services companies. In addition, periods of economic crisis and oil market instability are also found to have resulted in higher risk exposure in the US oil and gas stock returns. The findings comply with that of Manning (1991) who found changes in oil price to have a significant influence on UK oil and gas stocks. Elyasiani et al (2011) examined the association between oil price and stock returns using $\operatorname{GARCH}(1,1)$ which is a different technique from the conventional multifactor pricing model. Mohanty et al (2014) applied Fama-French-Carhart's four factor model augmented with changes in the oil price as an additional risk factor to the US travel and leisure industry. Oil risk exposure was found to be negative in most of the cases and vary considerably over gambling, hotels, airlines, restaurants, recreational services, travel and tourism. The impact of the oil price was found to be more significant on airlines, restaurants and bars and recreational services.

The most common methods used to study the relationship between oil price and oil companies' stock returns are co-integration analysis, multifactor regression model and volatility spill-over analysis (See Chou et al (2012) and Asteriou and Bashmakova (2013)).

\section{Data and Methodology}

\subsection{Data}

This study will cover all of the active oil and gas companies quoted on the main market of the London stock exchange. It includes oil and gas producers, oil equipment and services companies. The data consists of daily returns of oil and gas companies, the FTSE All Share index, the return (risk free rate) on UK Treasury 
bills and the Brent crude oil price for the period between January 2, 2004 and December 31, 2015. The Fama-French and momentum factors for UK stocks were adopted from Gregory et al (2013) constructed for the UK market.

\subsection{Methodology}

In this section, the four factor model of Fama-French-Carhart is augmented with the lagged returns of Brent crude oil price asymmetrically decomposed using Mork et al (1994) method into five lags each of oil price increases (+) and oil price decreases (-). Due to the nature and established characteristics of financial data, GARCH $(1,1)$ conditional variance specification is employed to estimate the following equation that represents the model used in the study.

$$
\begin{aligned}
R_{i t}-R_{f t} & =\alpha_{i 0}+\beta_{i m}\left(R_{m t}-R_{f t}\right)+\beta_{1} S M B_{t}+\beta_{2} H_{M L}+\beta_{3} \text { Mom }_{t} \\
& +\sum_{j=0}^{5} \beta_{\text {ioil } j_{j}}{ }^{+} R_{t} \text { OilPrice }_{t-j}{ }^{+}+\sum_{j=0}^{5} \beta_{\text {ioil }_{j}}{ }^{-} R_{t} \text { OilPrice }_{t-j}{ }^{-}+\varepsilon_{i t}
\end{aligned}
$$

Where $R_{i t}$ is company's daily stock return; $R_{f t}$ is the risk free rate of return (UK Treasury bill rate adjusted to a daily rate); $R_{i t}-R_{f t}$ is the individual stock's excess return; $\alpha_{i 0}$ is the constant; $\beta_{i m}$ is the coefficient representing the market risk (systematic risk) of the London stock exchange; $R_{m t}$ is market's expected daily returns; $R_{m t}-R_{f t}$ is the market's daily excess return (risk premium); $S M B_{t}$ is the Small Minus Big (Difference between the small and large stock portfolio returns based on companies' market values); $H M L_{t}$ is the High Minus Low (Difference between the high and low stock portfolio returns based on companies' book-tomarket values); $\mathrm{Mom}_{t}$ is the momentum factor (assumption that price is more likely to be moving in the same direction without change); $R_{t}$ OilPrice is the log 
changes of the Brent crude oil price decomposed into 5 lags of both positive and negative changes; and $\varepsilon_{i t}$ is the error term.

\section{Analysis and Results}

The following diagram shows the price level and fluctuation of the oil and gas sector, market index and oil price over the period January 2, 2004 to December $31,2015$.

Figure 1 Graphical Presentation of the Stock Market (FTSE All Share) Index, Oil and Gas Sector Index and Brent Crude Oil Price Daily Series

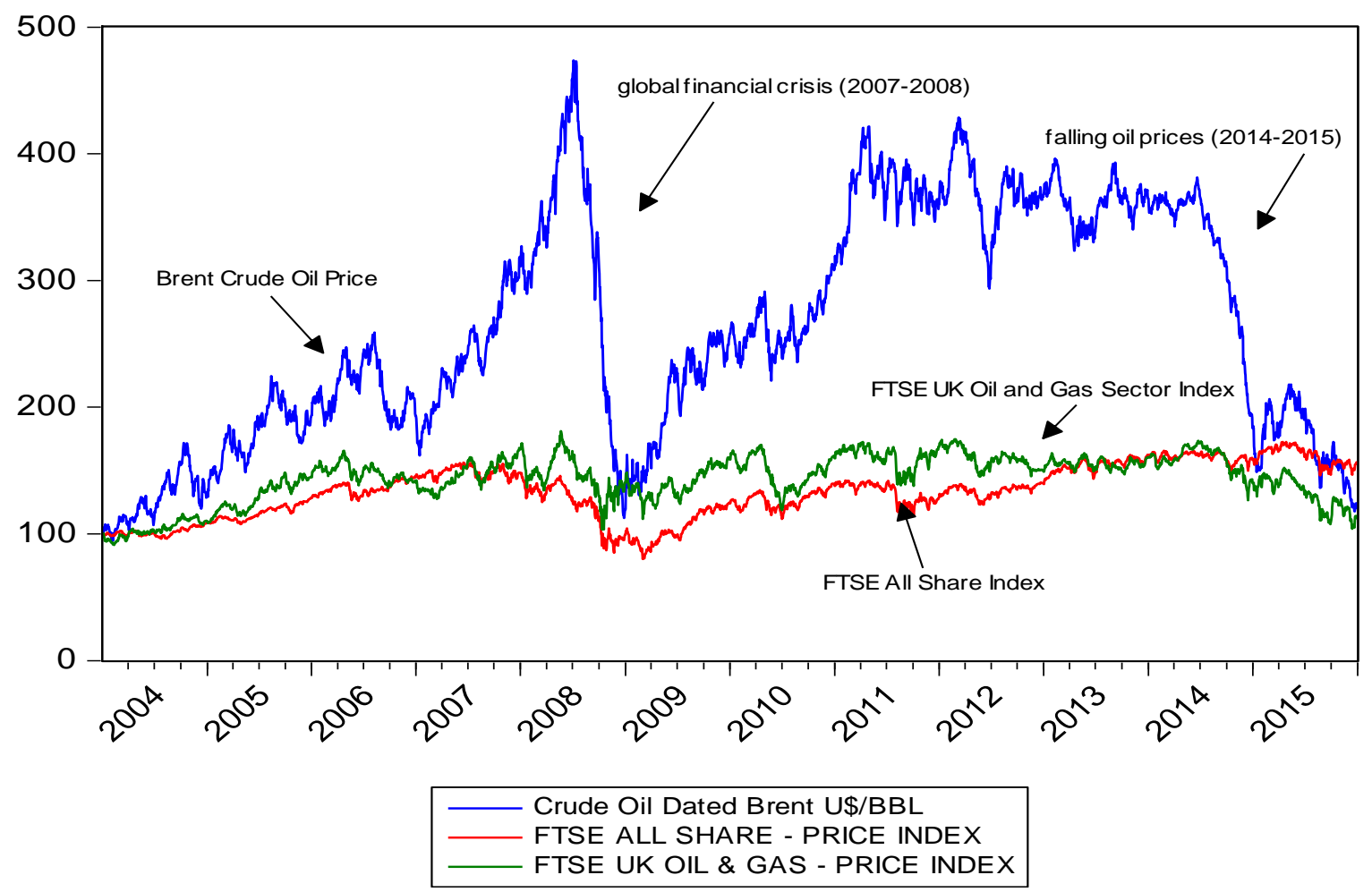

(Rebased: January 2, $2004=100)$

Figure 1 shows the relative performance and trends of the key parameters of the study; the Brent crude oil price, oil and gas sector, and the United Kingdom stock market index. The series are rebased to '100' at the same starting point of June 2, 2004 to December 31, 2015 in order to make effective comparative analysis. 
The Brent crude oil price is shown to be the most volatile series when compared to the market and oil sector indices. The shock or fall of the oil price between 2007 and 2008 was significant and thus could possibly be attributed to the effect of the global financial crisis (2007-2008). The oil price recovered to its position before the falling oil prices in 2014 through to 2015. The FTSE All Share and FTSE UK Oil and Gas Sector indices move in a similar direction and the series are more stable over the period than the oil price. One of the objective of using asset pricing model is to assess whether the observed high volatility in the Brent crude oil price has any impact on the valuation and pricing of oil and gas stocks quoted on the London stock exchange.

To address the two structural breaks observed in the Brent crude oil price of global financial crisis (2007-2008) and falling oil prices (2014-2015), dummy variables were introduced into the estimation of the parameters of the adopted model. Results are shown in Table 2.

Table 1 provides a summary of the descriptive statistics of the data showing the number of observations, mean, maximum and minimum values and standard deviation of monthly returns of every oil and gas stock included in the sample. 
Table 1 Summary Descriptive Statistics for the Oil and Gas Stocks' Daily Returns Between January 2, 2004 and December 31, 2015 (Full Sample)

\begin{tabular}{|c|c|c|c|c|c|c|}
\hline Company Name & $\begin{array}{c}\text { Country of } \\
\text { Main Business* }\end{array}$ & Obs. & Mean & Maximum & Minimum & Std. dev. \\
\hline Amec & Cheshire, UK & 3033 & 0.000165 & 0.135488 & -0.26344 & 0.020894 \\
\hline Aminex & Dublin, Ireland & 3033 & -0.000870 & 0.530628 & -0.52639 & 0.045603 \\
\hline BG Group & Reading, UK & 3033 & 0.000407 & 0.236238 & -0.14832 & 0.020536 \\
\hline $\mathrm{BP}$ & London, UK & 3033 & $-8.13 E-05$ & 0.105826 & -0.14037 & 0.016365 \\
\hline Cairn Energy & Edinburgh, UK & 3033 & 0.000409 & 0.400461 & -0.20008 & 0.025739 \\
\hline Dragon Oil & Dublin, Ireland & 3033 & 0.001057 & 0.174649 & -0.18540 & 0.026553 \\
\hline Fortune Oil & London, UK & 3033 & 0.000330 & 0.409156 & -0.18821 & 0.034637 \\
\hline Hunting & London, UK & 3033 & 0.000365 & 0.144006 & -0.10810 & 0.023849 \\
\hline JKX Oil and Gas & London, UK & 3033 & -0.00023 & 0.277032 & -0.29150 & 0.032834 \\
\hline Premier Oil & Edinburgh, UK & 3033 & -0.00021 & 0.158893 & -0.16045 & 0.026098 \\
\hline Royal Dutch Shell 'B' & London, UK & 3033 & $2.14 \mathrm{E}-05$ & 0.132139 & -0.09815 & 0.015776 \\
\hline Soco International & London, UK & 3033 & 0.000302 & 0.172966 & -0.42255 & 0.025738 \\
\hline Tullow Oil & London, UK & 3033 & 0.000217 & 0.216278 & -0.14079 & 0.026317 \\
\hline Wood Group (John) & Aberdeen, UK & 3033 & 0.000495 & 0.160203 & -0.14552 & 0.023864 \\
\hline Afren & London, UK & 2819 & -0.00086 & 1.038508 & -1.26243 & 0.053429 \\
\hline Hardy Oil and Gas & Douglas, Isle of Man & 2758 & -0.00077 & 0.257924 & -0.53430 & 0.033003 \\
\hline Royal Dutch Shell 'A' & London, UK & 2726 & $-5.37 \mathrm{E}-05$ & 0.128570 & -0.09789 & 0.015339 \\
\hline Petrofac & St. Helier, Jersey & 2673 & $5.19 \mathrm{E}-04$ & 0.128002 & -0.30715 & 0.025669 \\
\hline Lamprell & Douglas, Isle of Man & 2407 & -0.00019 & 0.186243 & -0.84213 & 0.041860 \\
\hline Salamander Energy & London, UK & 2371 & -0.00039 & 0.349839 & -0.24009 & 0.031404 \\
\hline Endeavor Intl. Corp & London, UK & 2099 & -0.00290 & 0.497156 & -3.15700 & 0.083248 \\
\hline Kentz & St. Helier, Jersey & 2063 & 0.001015 & 0.279463 & -0.10211 & 0.020190 \\
\hline Heritage Oil & St. Helier, Jersey & 2024 & 0.000213 & 0.220646 & -0.34245 & 0.030476 \\
\hline Cadogan Petroleum & Kyiv, Ukraine & 1967 & -0.00170 & 0.321584 & -0.38221 & 0.047489 \\
\hline Exillon Energy & Douglas, Isle of Man & 1576 & $-2.27 \mathrm{E}-04$ & 0.257829 & -0.23767 & 0.033476 \\
\hline Enquest & London, UK & 1498 & -0.00109 & 0.209721 & -0.16136 & 0.028271 \\
\hline Essar Energy & London, UK & 1477 & -0.00117 & 0.198245 & -0.30563 & 0.026204 \\
\hline Genel Energy & London, UK & 1185 & -0.00150 & 0.128019 & -0.13821 & 0.023801 \\
\hline Ophir Energy & London, UK & 1169 & -0.00067 & 0.172324 & -0.16101 & 0.02815 \\
\hline Ruspetro & London, UK & 1031 & -0.00297 & 0.35065 & -0.28377 & 0.045921 \\
\hline
\end{tabular}

* Country of main business is defined by the registered office (country of incorporation) of the companies. Oil and gas companies operate in various countries of the world especially where oil and gas are found.

\subsection{Fama-French-Carhart's Four Factor Asset Pricing Model Augmented with International Oil Price}

The following table shows the coefficients of the multivariate regression model developed using Fama- French-Carhart's (1997) four factor asset pricing model plus the oil price as additional risk factor. It also shows the effect of global financial crisis (2007-2008) and falling oil prices (2014-2015) considered as structural breaks in the model. 
Table 2 Fama-French-Carhart's Four Factor Asset Pricing Model Augmented with Brent Crude Oil Price

\begin{tabular}{|c|c|c|c|c|c|c|c|c|c|c|c|}
\hline Coefficients: & Amec & Aminex & BG Group & BP & $\begin{array}{l}\text { Cairn } \\
\text { Energy }\end{array}$ & Dragon Oil & Fortune Oil & Hunting & $\begin{array}{l}\text { JKX Oil and } \\
\text { Gas }\end{array}$ & Premier Oil & $\begin{array}{l}\text { Royal Dutch } \\
\text { Shell 'B' }\end{array}$ \\
\hline \multirow[t]{2}{*}{ Intercept $\left(\alpha_{i 0}\right)$} & -0.000057 & -0.001721 & -0.000254 & -0.000231 & -0.000453 & 0.000203 & -0.000471 & 0.000148 & $-0.0011 * *$ & -0.000408 & -0.000188 \\
\hline & $(0.8472)$ & $(0.0594)$ & $(0.7438)$ & $(0.2667)$ & $(0.2177)$ & $(0.6061)$ & $(0.4417)$ & $(0.6931)$ & $(0.0214)$ & $(0.2253)$ & $(0.3262)$ \\
\hline \multirow[t]{2}{*}{ Market $\left(\beta_{i, m}\right)$} & $1.049831^{* * *}$ & $0.5889 * * *$ & $0.9798 * * *$ & $0.7758 * * *$ & $1.1304 * * *$ & $0.6979 * * *$ & $0.5505^{* * *}$ & $1.1108^{* * *}$ & $1.0445 * * *$ & $1.0503 * * *$ & $0.8009 * * *$ \\
\hline & $(0.0000)$ & $(0.0000)$ & $(0.0000)$ & $(0.0000)$ & $(0.0000)$ & $(0.0000)$ & $(0.0000)$ & $(0.0000)$ & $(0.0000)$ & $(0.0000)$ & $(0.0000)$ \\
\hline \multirow[t]{2}{*}{$\operatorname{SMB}\left(\beta_{1}\right)$} & $0.189704 * * *$ & $1.0651^{* * *}$ & $-0.235^{* * *}$ & $-0.451 * * *$ & $0.2790^{* * *}$ & $0.2668 * * *$ & $0.5646 * * *$ & $0.7124 * * *$ & $0.7985^{* * *}$ & $0.3939 * * *$ & $-0.471 * * *$ \\
\hline & $(0.0000)$ & $(0.0000)$ & $(0.0079)$ & $(0.0000)$ & $(0.0000)$ & $(0.0000)$ & $(0.0000)$ & $(0.0000)$ & $(0.0000)$ & $(0.0000)$ & $(0.0000)$ \\
\hline \multirow[t]{2}{*}{$\mathrm{HML}\left(\beta_{2}\right)$} & $0.256515^{* * *}$ & -0.12826 & 0.093795 & $0.1603 * * *$ & -0.022543 & $0.1846 * * *$ & 0.088579 & $0.1440 * *$ & $-0.1590 * *$ & $0.1980 * * *$ & $0.0970 * * *$ \\
\hline & $(0.0000)$ & $(0.2699)$ & $(0.4099)$ & $(0.0000)$ & $(0.7304)$ & $(0.0022)$ & $(0.4215)$ & $(0.0385)$ & $(0.0477)$ & $(0.0051)$ & $(0.0024)$ \\
\hline \multirow[t]{2}{*}{ Mom $\left(\beta_{3}\right)$} & $-0.09151 * * *$ & $0.1719 * * *$ & -0.106503 & $-0.064 * * *$ & $-0.112^{* * *}$ & -0.060741 & $-0.185^{* * *}$ & $-0.166^{* * *}$ & -0.03897 & -0.012693 & -0.018638 \\
\hline & $(0.0069)$ & $(0.002)$ & $(0.0697)$ & $(0.0023)$ & $(0.0069)$ & $(0.2181)$ & $(0.0044)$ & $(0.0000)$ & $(0.4971)$ & $(0.755)$ & $(0.3335)$ \\
\hline \multirow[t]{2}{*}{ Brent crude oil $\left(\beta_{4}\right)$} & $0.063195 * * *$ & $0.0785^{* * *}$ & $0.1614 * * *$ & $0.1170 * * *$ & $0.1701 * * *$ & $0.1445^{* * *}$ & 0.019164 & $0.1242 * * *$ & $0.1215^{* * *}$ & $0.1917 * * *$ & $0.0983^{* * *}$ \\
\hline & $(0.0000)$ & $(0.0071)$ & $(0.0000)$ & $(0.0000)$ & $(0.0000)$ & $(0.0000)$ & $(0.4693)$ & $(0.0000)$ & $(0.0000)$ & $(0.0000)$ & $(0.0000)$ \\
\hline \multirow{4}{*}{ 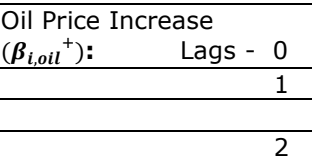 } & $\begin{array}{c}0.062117^{* *} \\
(0.0215)\end{array}$ & $\begin{array}{c}0.19472 * * \\
(0.0235)\end{array}$ & $\begin{array}{c}0.1566 * * * \\
(0.0000)\end{array}$ & $\begin{array}{c}0.0748 * * * \\
(0.0001)\end{array}$ & $\begin{array}{c}0.2151 * * * \\
(0.0000)\end{array}$ & $\begin{array}{l}0.1316 * * * \\
(0.0014)\end{array}$ & $\begin{array}{c}-0.093183 \\
(0.1119)\end{array}$ & $\begin{array}{c}0.1510 * * * \\
(0.0000)\end{array}$ & $\begin{array}{c}0.1996 * * * \\
(0.0000)\end{array}$ & $\begin{array}{c}0.1600 * * * \\
(0.0000)\end{array}$ & $\begin{array}{c}0.0751 * * * \\
(0.0000)\end{array}$ \\
\hline & -0.01702 & -0.078024 & $0.0736 * * *$ & 0.026655 & $0.0926 * * *$ & 0.059898 & -0.023501 & $0.1362 * * *$ & 0.00926 & $0.1400 * * *$ & -0.00547 \\
\hline & $(0.5258)$ & $(0.3718)$ & $(0.0024)$ & $(0.1267)$ & $(0.0001)$ & $(0.0970)$ & $(0.6773)$ & $(0.0004)$ & $(0.8625)$ & $(0.0001)$ & $(0.7601)$ \\
\hline & 0.013135 & 0.138944 & -0.00132 & -0.013437 & $-0.089 * * *$ & -0.010493 & $0.1262 * *$ & -0.019697 & 0.062237 & 0.013992 & -0.01501 \\
\hline 2 & $(0.6183)$ & $(0.1584)$ & $(0.9625)$ & $(0.4289)$ & $(0.0047)$ & $(0.7615)$ & $(0.0184)$ & $(0.5923)$ & $(0.1618)$ & $(0.7115)$ & $(0.4229)$ \\
\hline \multirow[t]{2}{*}{3} & -0.007929 & -0.021425 & 0.008753 & -0.003272 & -0.062386 & -0.0123 & 0.00918 & -0.055283 & -0.04272 & -0.003001 & 0.007022 \\
\hline & $(0.7679)$ & $(0.8492)$ & $(0.7679)$ & $(0.8636)$ & $(0.0875)$ & $(0.7187)$ & $(0.8892)$ & $(0.1458)$ & $(0.3923)$ & $(0.9252)$ & $(0.7112)$ \\
\hline \multirow[t]{2}{*}{4} & -0.02638 & 0.045853 & -0.011439 & 0.008433 & 0.044309 & -0.03085 & -0.047893 & 0.009867 & 0.028372 & -0.012597 & -0.006269 \\
\hline & $(0.324)$ & $(0.6723)$ & $(0.6578)$ & $(0.6398)$ & $(0.1779)$ & $(0.3881)$ & $(0.3588)$ & $(0.803)$ & $(0.5567)$ & $(0.7193)$ & $(0.7513)$ \\
\hline \multirow[t]{2}{*}{5} & 0.025 & -0.08279 & -0.025483 & -0.002485 & 0.040296 & -0.068425 & 0.005559 & 0.008893 & -0.016398 & 0.008844 & -0.009217 \\
\hline & $(0.3569)$ & $(0.268)$ & $(0.3053)$ & $(0.9000)$ & $(0.1696)$ & $(0.0721)$ & $(0.9249)$ & $(0.8125)$ & $(0.7006)$ & $(0.8260)$ & $(0.6006)$ \\
\hline \multirow{2}{*}{ 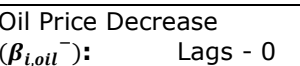 } & 0.044597 & 0.062485 & $0.1890 * * *$ & $0.1390 * * *$ & $0.1167 * * *$ & $0.1390 * * *$ & $0.1631 * * *$ & $0.1631 * * *$ & $0.1795^{* * *}$ & $0.2212^{* * *}$ & -0.002404 \\
\hline & $(0.1231)$ & $(0.4311)$ & $(0.0000)$ & $(0.0000)$ & $(0.0024)$ & $(0.0002)$ & $(0.0089)$ & $(0.0089)$ & $(0.0007)$ & $(0.0000)$ & $(0.9269)$ \\
\hline \multirow[t]{2}{*}{1} & 0.034681 & $0.2625^{* * *}$ & -0.008648 & -0.01201 & 0.055732 & -0.035611 & 0.047216 & 0.047216 & 0.062322 & $0.0760 * *$ & 0.029886 \\
\hline & $(0.234)$ & $(0.0002)$ & $(0.7878)$ & $(0.5177)$ & $(0.1305)$ & $(0.3676)$ & $(0.3228)$ & $(0.3228)$ & $(0.2332)$ & $(0.0163)$ & $(0.3153)$ \\
\hline \multirow[t]{2}{*}{2} & 0.043789 & -0.095608 & 0.023334 & 0.008063 & $-1.57 \mathrm{E}-05$ & 0.041638 & 0.078608 & 0.078608 & 0.021385 & 0.017333 & -0.006048 \\
\hline & $(0.1275)$ & $(0.1903)$ & $(0.4800)$ & $(0.6540)$ & $(0.9997)$ & $(0.2122)$ & $(0.1701)$ & $(0.1701)$ & $(0.6669)$ & $(0.6127)$ & $(0.8294)$ \\
\hline \multirow[t]{2}{*}{3} & -0.030804 & -0.073433 & -0.059667 & -0.022554 & -0.024779 & 0.018286 & 0.006868 & 0.006868 & 0.041357 & -0.004415 & 0.03292 \\
\hline & $(0.3218)$ & $(0.3073)$ & $(0.0600)$ & $(0.2215)$ & $(0.4905)$ & $(0.6376)$ & $(0.9114)$ & $(0.9114)$ & $(0.4495)$ & $(0.9086)$ & $(0.2296)$ \\
\hline \multirow[t]{2}{*}{4} & 0.007021 & -0.00112 & -0.026628 & 0.023444 & -0.015578 & 0.028939 & $-0.1356 * *$ & $-0.1356 * *$ & $-0.1036 * *$ & 0.00813 & -0.054889 \\
\hline & $(0.8203)$ & $(0.9868)$ & $(0.4188)$ & $(0.2111)$ & $(0.6776)$ & $(0.4075)$ & $(0.0226)$ & $(0.0226)$ & $(0.042)$ & $(0.8242)$ & $(0.0581)$ \\
\hline \multirow[t]{2}{*}{5} & 0.031142 & 0.050511 & -0.021953 & 0.002021 & -0.035941 & -0.030318 & $-0.153 * * *$ & $-0.153 * * *$ & -0.011185 & 0.022397 & -0.010468 \\
\hline & $(0.3136)$ & $(0.5165)$ & $(0.5046)$ & $(0.9209)$ & $(0.3648)$ & $(0.4325)$ & $(0.0032)$ & $(0.0032)$ & $(0.8283)$ & $(0.5446)$ & $(0.7033)$ \\
\hline $\begin{array}{l}\text { Dummy } 1 \text { - global } \\
\text { financial crisis ( } 2007- \\
2008 \text { ) }\end{array}$ & $\begin{array}{c}0.001762 * * \\
(0.0131)\end{array}$ & $\begin{array}{l}0.000254 \\
(0.9174)\end{array}$ & $\begin{array}{c}0.001429 \\
(0.3066)\end{array}$ & $\begin{array}{c}-0.000008 \\
(0.9855)\end{array}$ & $\begin{array}{l}0.001597 \\
(0.0814)\end{array}$ & $\begin{array}{l}0.000688 \\
(0.5250)\end{array}$ & $\begin{array}{c}0.000243 \\
(0.8745)\end{array}$ & $\begin{array}{l}0.001451 \\
(0.0871)\end{array}$ & $\begin{array}{c}0.00289 * * \\
(0.0170)\end{array}$ & $\begin{array}{l}0.000579 \\
(0.5168)\end{array}$ & $\begin{array}{c}0.0000668 \\
(0.8858)\end{array}$ \\
\hline $\begin{array}{l}\text { Dummy } 2 \text { - falling oil } \\
\text { prices }(2014-2015)\end{array}$ & $\begin{array}{c}-0.001327 \\
(0.1176) \\
\end{array}$ & $\begin{array}{c}-0.000594 \\
(0.8384) \\
\end{array}$ & $\begin{array}{l}0.000276 \\
(0.8958) \\
\end{array}$ & $\begin{array}{c}0.0000447 \\
(0.9394)\end{array}$ & $\begin{array}{l}0.001091 \\
(0.3509) \\
\end{array}$ & $\begin{array}{l}0.000079 \\
(0.9389) \\
\end{array}$ & $\begin{array}{l}0.000334 \\
(0.8294) \\
\end{array}$ & $\begin{array}{c}-0.001171 \\
(0.326) \\
\end{array}$ & $\begin{array}{c}-0.001674 \\
(0.2467) \\
\end{array}$ & $\begin{array}{c}-0.000673 \\
(0.5857) \\
\end{array}$ & $\begin{array}{l}-0.00059 \\
(0.2551) \\
\end{array}$ \\
\hline
\end{tabular}




\begin{tabular}{|c|c|c|c|c|c|c|c|c|c|c|c|}
\hline Coefficients: & $\begin{array}{c}\text { Soco } \\
\text { International }\end{array}$ & Tullow Oil & $\begin{array}{l}\text { Wood Group } \\
\text { (John) }\end{array}$ & Afren & $\begin{array}{l}\text { Hardy Oil } \\
\text { and Gas }\end{array}$ & $\begin{array}{l}\text { Royal Dutch } \\
\text { Shell 'A' }\end{array}$ & Petrofac & Lamprell & $\begin{array}{c}\text { Salamander } \\
\text { Energy }\end{array}$ & $\begin{array}{c}\text { Endeavor } \\
\text { International } \\
\text { Corp. }\end{array}$ & Kentz \\
\hline \multirow[t]{2}{*}{ Intercept $\left(\alpha_{i 0}\right)$} & $-0.00081 * *$ & -0.000172 & 0.0000348 & 0.000141 & -0.000185 & 0.000123 & $0.00121 * *$ & $0.0026 * * *$ & $0.00056 * *$ & -0.001113 & 0.00148 \\
\hline & $(0.0177)$ & $(0.6483)$ & $(0.9214)$ & $(0.8082)$ & $(0.7815)$ & $(0.6324)$ & $(0.0461)$ & $(0.0000)$ & $(0.017)$ & $(0.8317)$ & $(0.2144)$ \\
\hline \multirow{2}{*}{ Market $\left(\beta_{i, m}\right)$} & $0.9680 * * *$ & $1.1746 * * *$ & $1.1871 * * *$ & $1.2279 * * *$ & -0.031562 & 0.018143 & 0.090281 & 0.000010 & 0.0000163 & -0.086004 & 0.010152 \\
\hline & $(0.0000)$ & $(0.0000)$ & $(0.0000)$ & $(0.0000)$ & $(0.6353)$ & $(0.5045)$ & $(0.1033)$ & $(0.7037)$ & $(0.6364)$ & $(0.9039)$ & $(0.9218)$ \\
\hline \multirow[t]{2}{*}{$\operatorname{SMB}\left(\beta_{1}\right)$} & $0.4570 * * *$ & $0.1434 * *$ & $0.3920 * * *$ & $0.6251^{* * *}$ & -0.02584 & 0.068747 & 0.034352 & 0.0000132 & 0.0000357 & -0.24282 & -0.011756 \\
\hline & $(0.0000)$ & $(0.0145)$ & $(0.0000)$ & $(0.0000)$ & $(0.7671)$ & $(0.1132)$ & $(0.6479)$ & $(0.7594)$ & $(0.5077)$ & $(0.7124)$ & $(0.9488)$ \\
\hline \multirow[t]{2}{*}{ HML $\left(\beta_{2}\right)$} & $-0.13512 * *$ & $-0.170 * * *$ & $0.2663 * * *$ & 0.123332 & -0.078311 & -0.000298 & -0.171472 & 0.0000043 & 0.0000242 & 0.003631 & 0.029983 \\
\hline & $(0.0113)$ & $(0.0097)$ & $(0.0001)$ & $(0.2808)$ & $(0.5517)$ & $(0.9952)$ & $(0.0815)$ & $(0.8939)$ & $(0.599)$ & $(0.9962)$ & $(0.8786)$ \\
\hline \multirow[t]{2}{*}{ Mom $\left(\beta_{3}\right)$} & 0.045721 & $-0.116 * * *$ & $-0.169 * * *$ & -0.01093 & -0.037816 & 0.039725 & -0.01578 & 0.0000103 & 0.0000194 & -0.100731 & 0.05128 \\
\hline & $(0.294)$ & $(0.0068)$ & $(0.0000)$ & $(0.8625)$ & $(0.6105)$ & $(0.2234)$ & $(0.7926)$ & $(0.8271)$ & $(0.719)$ & $(0.8878)$ & $(0.6600)$ \\
\hline \multirow[t]{2}{*}{ Brent crude oil $\left(\beta_{4}\right)$} & $0.1368 * * *$ & $0.2450 * * *$ & $0.2012 * * *$ & $0.2146 * * *$ & -0.012388 & -0.00392 & 0.00842 & -0.000002 & -0.0000045 & -0.019469 & -0.007309 \\
\hline & $(0.0000)$ & $(0.0000)$ & $(0.0000)$ & $(0.0000)$ & $(0.7084)$ & $(0.7561)$ & $(0.7412)$ & $(0.8075)$ & $(0.637)$ & $(0.926)$ & $(0.8881)$ \\
\hline \multirow{2}{*}{$\begin{array}{lr}\begin{array}{l}\text { Oil Price Increase } \\
\left(\boldsymbol{\beta}_{i, o i l}{ }^{+}\right):\end{array} & \text {Lags - } 0 \\
& 1 \\
\end{array}$} & $\begin{array}{c}0.2606 * * * \\
(0.0000)\end{array}$ & $\begin{array}{c}0.2949 * * * \\
(0.0000)\end{array}$ & $\begin{array}{c}0.1311^{* * *} \\
(0.0003)\end{array}$ & $\begin{array}{c}0.2120 * * * \\
(0.0003)\end{array}$ & $\begin{array}{l}0.117707 \\
(0.0665) \\
\end{array}$ & $\begin{array}{c}-0.0587 * * \\
(0.0326)\end{array}$ & $\begin{array}{l}0.076475 \\
(0.2641)\end{array}$ & $\begin{array}{l}-0.001087 \\
(0.2434)\end{array}$ & $\begin{array}{l}0.071475 \\
(0.3072)\end{array}$ & $\begin{array}{l}0.217367 \\
(0.8297)\end{array}$ & $\begin{array}{l}-0.041133 \\
(0.3725)\end{array}$ \\
\hline & $0.0813^{* *}$ & 0.081232 & 0.020392 & 0.078036 & $0.12508^{* *}$ & 0.046565 & -0.030795 & -0.001627 & 0.034377 & -0.217202 & -0.019866 \\
\hline & $(0.0104)$ & $(0.0175)$ & $(0.5241)$ & $(0.0366)$ & $(0.0207)$ & $(0.1004)$ & $(0.5512)$ & $(0.0723)$ & $(0.5408)$ & $(0.7888)$ & $(0.6709)$ \\
\hline \multirow[t]{2}{*}{2} & $-0.1025 * * *$ & -0.00427 & -0.005525 & -0.062276 & -0.003766 & -0.05291 & $0.1212^{* *}$ & $-0.0019 * *$ & -0.000109 & 0.022435 & -0.01161 \\
\hline & $(0.0001)$ & $(0.9125)$ & $(0.8414)$ & $(0.1663)$ & $(0.9570)$ & $(0.0784)$ & $(0.0424)$ & $(0.0499)$ & $(0.9987)$ & $(0.9818)$ & $(0.8181)$ \\
\hline \multirow[t]{2}{*}{3} & $-0.0891 * * *$ & -0.016381 & 0.001533 & 0.094514 & -0.080444 & 0.029921 & 0.013371 & $-0.002 * * *$ & 0.000483 & 0.328664 & -0.020667 \\
\hline & $(0.0005)$ & $(0.6972)$ & $(0.9641)$ & $(0.0618)$ & $(0.1759)$ & $(0.2995)$ & $(0.7943)$ & $(0.0036)$ & $(0.9941)$ & $(0.7142)$ & $(0.6817)$ \\
\hline \multirow[t]{2}{*}{4} & 0.045631 & 0.044144 & 0.014878 & -0.005827 & -0.059872 & -0.003052 & 0.010435 & $-0.001 * * *$ & 0.028318 & 0.081436 & 0.046297 \\
\hline & $(0.0962)$ & $(0.2987)$ & $(0.6655)$ & $(0.9024)$ & $(0.2608)$ & $(0.9114)$ & $(0.8320)$ & $(0.0085)$ & $(0.6795)$ & $(0.9524)$ & $(0.3486)$ \\
\hline \multirow[t]{2}{*}{5} & -0.029619 & -0.042828 & 0.051211 & $-0.202^{* * *}$ & -0.00204 & 0.022867 & -0.008549 & -0.000952 & -0.024084 & -0.093378 & 0.095427 \\
\hline & $(0.3461)$ & $(0.2940)$ & $(0.1424)$ & $(0.0002)$ & $(0.9780)$ & $(0.3809)$ & $(0.8816)$ & $(0.2805)$ & $(0.6495)$ & $(0.9393)$ & $(0.0643)$ \\
\hline $\begin{array}{l}\text { Oil Price Decrease } \\
\left(\boldsymbol{\beta}_{i, \text { oil }}\right): \quad \text { Lags - } 0 \\
\end{array}$ & $\begin{array}{l}0.1311 * * * \\
(0.0014)\end{array}$ & $\begin{array}{c}0.2330 * * * \\
(0.0000)\end{array}$ & $\begin{array}{c}0.2153 * * * \\
(0.0000)\end{array}$ & $\begin{array}{c}0.2884 * * * \\
(0.0000)\end{array}$ & $\begin{array}{l}0.044597 \\
(0.1231)\end{array}$ & $\begin{array}{l}-0.00240 \\
(0.9269)\end{array}$ & $\begin{array}{l}0.008307 \\
(0.8510)\end{array}$ & $\begin{array}{l}0.009069 \\
(0.1833)\end{array}$ & $\begin{array}{l}0.010997 \\
(0.7680)\end{array}$ & $\begin{array}{l}0.120593 \\
(0.5020)\end{array}$ & $\begin{array}{c}0.0000229 \\
(0.0599)\end{array}$ \\
\hline \multirow[t]{2}{*}{1} & $0.0847 * *$ & $-0.120 * * *$ & 0.027856 & -0.006105 & 0.034681 & 0.029886 & $-0.1024 * *$ & 0.011292 & -0.013173 & -0.104195 & $0.00001^{* *}$ \\
\hline & $(0.0299)$ & $(0.0001)$ & $(0.4275)$ & $(0.9234)$ & $(0.2340)$ & $(0.3153)$ & $(0.0298)$ & $(0.0781)$ & $(0.7442)$ & $(0.6290)$ & $(0.0409)$ \\
\hline \multirow[t]{2}{*}{2} & -0.020135 & 0.06194 & -0.036484 & $-0.249 * * *$ & 0.043789 & -0.006048 & $-0.0900 * *$ & $0.0101 * *$ & $-0.04895^{* *}$ & -0.01436 & $0.0002^{* * *}$ \\
\hline & $(0.5669)$ & $(0.1132)$ & $(0.2460)$ & $(0.0000)$ & $(0.1275)$ & $(0.8294)$ & $(0.0430)$ & $(0.0159)$ & $(0.0242)$ & $(0.9591)$ & $(0.0084)$ \\
\hline \multirow[t]{2}{*}{3} & -0.014661 & -0.055759 & -0.048591 & 0.001057 & -0.030804 & 0.03292 & $0.09651 * *$ & 0.010927 & -0.021017 & -0.069305 & 0.0000155 \\
\hline & $(0.6986)$ & $(0.0879)$ & $(0.2021)$ & $(0.9900)$ & $(0.3218)$ & $(0.2296)$ & $(0.0479)$ & $(0.1028)$ & $(0.4926)$ & $(0.8016)$ & $(0.0709)$ \\
\hline \multirow[t]{2}{*}{4} & -0.04072 & -0.038818 & 0.021931 & 0.082577 & 0.007021 & -0.054889 & -0.05812 & $0.0165 * * *$ & -0.005785 & 0.041768 & 0.0000258 \\
\hline & $(0.3109)$ & $(0.1800)$ & $(0.5007)$ & $(0.2200)$ & $(0.8203)$ & $(0.0581)$ & $(0.2008)$ & $(0.0008)$ & $(0.8403)$ & $(0.8655)$ & $(0.0125)$ \\
\hline \multirow[t]{2}{*}{5} & 0.007037 & 0.016704 & 0.025163 & 0.009215 & 0.031142 & -0.010468 & -0.052178 & 0.006788 & 0.007636 & 0.038328 & $0.0002 * * *$ \\
\hline & $(0.8467)$ & $(0.6247)$ & $(0.4684)$ & $(0.8927)$ & $(0.3136)$ & $(0.7033)$ & $(0.2251)$ & $(0.1653)$ & $(0.8215)$ & $(0.8447)$ & $(0.0064)$ \\
\hline $\begin{array}{l}\text { Dummy1 - global } \\
\text { financial crisis (2007- } \\
\text { 2008) }\end{array}$ & $\begin{array}{l}0.000517 \\
(0.5159) \\
\end{array}$ & $\begin{array}{l}0.000756 \\
(0.4094) \\
\end{array}$ & $\begin{array}{c}0.000962 \\
(0.284)\end{array}$ & $\begin{array}{l}0.00226 \\
(0.1135) \\
\end{array}$ & $\begin{array}{l}0.000842 \\
(0.5709) \\
\end{array}$ & $\begin{array}{l}0.000696 \\
(0.3222) \\
\end{array}$ & $\begin{array}{l}0.000526 \\
(0.6689)\end{array}$ & $\begin{array}{l}0.000684 \\
(0.5324) \\
\end{array}$ & $\begin{array}{c}-0.0000259 \\
(0.9775) \\
\end{array}$ & $\begin{array}{c}0.0004696 \\
(0.4222)\end{array}$ & $\begin{array}{c}-0.001825 \\
(0.5119) \\
\end{array}$ \\
\hline $\begin{array}{l}\text { Dummy } 2 \text { - falling oil } \\
\text { prices }(2014-2015)\end{array}$ & $\begin{array}{c}0.00395^{* * *} \\
(0.0001)\end{array}$ & $\begin{array}{c}-0.0026^{* *} \\
(0.033)\end{array}$ & $\begin{array}{c}-0.000517 \\
(0.6159) \\
\end{array}$ & $\begin{array}{c}-0.021 * * * \\
(0.0000)\end{array}$ & $\begin{array}{c}-0.0048^{* *} \\
(0.015)\end{array}$ & $\begin{array}{l}-0.000687 \\
(0.3222) \\
\end{array}$ & $\begin{array}{c}-0.007 * * * \\
(0.0000)\end{array}$ & $\begin{array}{c}-0.002 * * * \\
(0.0000) \\
\end{array}$ & $\begin{array}{c}-0.00057 * * \\
(0.0141)\end{array}$ & $\begin{array}{l}0.000696 \\
(0.2222) \\
\end{array}$ & $\begin{array}{c}-0.000716 \\
(0.8735)\end{array}$ \\
\hline & & & & & & & & & & & \\
\hline & & & & & & & & & & & \\
\hline
\end{tabular}




\begin{tabular}{|c|c|c|c|c|c|c|c|c|c|}
\hline Coefficients: & Heritage Oil & $\begin{array}{l}\text { Cadogan } \\
\text { Petroleum }\end{array}$ & $\begin{array}{l}\text { Exillon } \\
\text { Energy }\end{array}$ & Enquest & $\begin{array}{l}\text { Essar } \\
\text { Energy }\end{array}$ & $\begin{array}{l}\text { Genel } \\
\text { Energy }\end{array}$ & $\begin{array}{l}\text { Ophir } \\
\text { Energy }\end{array}$ & Ruspetro & \\
\hline Intercept $\left(\alpha_{i 0}\right)$ & $-0.00001 * * *$ & -0.000837 & $-0.001 * * *$ & $-0.003 * * *$ & $-0.001 * * *$ & $-0.002 * * *$ & 0.000790 & $-0.004 * * *$ & \\
\hline & $(0.0000)$ & $(0.2596)$ & $(0.0000)$ & $(0.0062)$ & $(0.0000)$ & $(0.0000)$ & $(0.4042)$ & $(0.0058)$ & \\
\hline Market $\left(\beta_{i, m}\right)$ & -0.0000027 & -0.095971 & 0.0000256 & 0.0000265 & -0.000006 & -0.000002 & -0.092171 & $0.4685^{* * *}$ & \\
\hline & $(0.8269)$ & $(0.2256)$ & $(0.1349)$ & $(0.1282)$ & $(0.9446)$ & $(0.8801)$ & $(0.3941)$ & $(0.0341)$ & \\
\hline SMB $\left(\beta_{1}\right)$ & -0.0000057 & 0.008662 & 0.0000496 & 0.0000451 & -0.000017 & 0.000021 & -0.011111 & 0.572243 & \\
\hline & $(0.7691)$ & $(0.9358)$ & $(0.0515)$ & $(0.0745)$ & $(0.141)$ & $(0.3022)$ & $(0.9520)$ & $(0.0521)$ & \\
\hline $\mathrm{HML}\left(\beta_{2}\right)$ & 0.0000105 & 0.071031 & -0.000007 & -0.000003 & -0.000027 & 0.0000035 & -0.076232 & 0.21522 & \\
\hline & $(0.4517)$ & $(0.5758)$ & $(0.6651)$ & $(0.8452)$ & $(0.0886)$ & $(0.8515)$ & $(0.7189)$ & $(0.5099)$ & \\
\hline Mom $\left(\beta_{3}\right)$ & 0.0000091 & 0.084956 & 0.0000079 & 0.0000091 & -0.000018 & -0.000008 & 0.033879 & -0.11206 & \\
\hline & $(0.5297)$ & $(0.3583)$ & $(0.5085)$ & $(0.4266)$ & $(0.1279)$ & $(0.4047)$ & $(0.8013)$ & $(0.7036)$ & \\
\hline Brent crude oil $\left(\beta_{4}\right)$ & -0.0000062 & -0.050457 & 0.0000038 & 0.0000026 & -0.000005 & 0.0000034 & 0.00611 & 0.041006 & \\
\hline & $(0.1607)$ & $(0.1055)$ & $(0.4009)$ & $(0.5634)$ & $(0.3473)$ & $(0.4683)$ & $(0.9125)$ & $(0.6705)$ & \\
\hline $\begin{array}{l}\text { Oil Price Increase } \\
\left(\boldsymbol{\beta}_{\text {ioil }}^{+}\right): \quad \text { Lags }-0\end{array}$ & $\begin{array}{l}-0.0000106 \\
(0.3606)\end{array}$ & $\begin{array}{l}0.143125 \\
(0.0649)\end{array}$ & $\begin{array}{c}0.0040 * * * \\
(0.0011)\end{array}$ & $\begin{array}{c}0.0000016 \\
(0.8592)\end{array}$ & $\begin{array}{l}-0.0001 * * \\
(0.0485)\end{array}$ & $\begin{array}{c}0.0452 * * * \\
(0.0000)\end{array}$ & $\begin{array}{c}-0.008726 \\
(0.941)\end{array}$ & $\begin{array}{l}-0.04053 \\
(0.8653)\end{array}$ & \\
\hline 1 & -0.0000208 & -0.084118 & $0.0044 * * *$ & -0.000015 & $-0.002 * * *$ & $0.0406 * * *$ & 0.05987 & -0.182724 & \\
\hline & $(0.0638)$ & $(0.2374)$ & $(0.0002)$ & $(0.1365)$ & $(0.0015)$ & $(0.0000)$ & $(0.6914)$ & $(0.3827)$ & \\
\hline 2 & -0.0000148 & -0.047498 & $0.0063 * * *$ & -0.000009 & $-0.002 * * *$ & $0.0566 * * *$ & $0.3065 * *$ & 0.280748 & \\
\hline & $(0.0975)$ & $(0.5437)$ & $(0.0000)$ & $(0.275)$ & $(0.0053)$ & $(0.0000)$ & $(0.0159)$ & $(0.3182)$ & \\
\hline 3 & -0.0000044 & -0.034485 & $0.0063 * * *$ & 0.0000037 & $-0.001 * * *$ & $0.0571 * * *$ & -0.196221 & -0.025243 & \\
\hline & $(0.5085)$ & $(0.6957)$ & $(0.0000)$ & $(0.605)$ & $(0.1108)$ & $(0.0000)$ & $(0.1088)$ & $(0.9204)$ & \\
\hline 4 & $-0.00002 * * *$ & 0.027965 & $0.0042 * * *$ & -0.000013 & $-0.002 * * *$ & $0.0362 * * *$ & 0.097197 & 0.267571 & \\
\hline & $(0.0000)$ & $(0.7584)$ & $(0.0000)$ & $(0.0806)$ & $(0.0002)$ & $(0.0000)$ & $(0.4001)$ & $(0.3237)$ & \\
\hline 5 & -0.0000128 & -0.115597 & $0.00292 * *$ & -0.000004 & -0.00001 & $0.0140 * * *$ & 0.050866 & 0.043296 & \\
\hline & $(0.2125)$ & $(0.1487)$ & $(0.0251)$ & $(0.5518)$ & $(0.0659)$ & $(0.1419)$ & $(0.7013)$ & $(0.8512)$ & \\
\hline $\begin{array}{l}\text { Oil Price Decrease } \\
\left(\boldsymbol{\beta}_{\text {ioil }}{ }^{-}\right): \quad \text { Lags - } 0\end{array}$ & $\begin{array}{c}0.0000138 \\
(0.2412)\end{array}$ & $\begin{array}{l}-0.119536 \\
(0.0802)\end{array}$ & $\begin{array}{l}0.0000095 \\
(0.2944)\end{array}$ & $\begin{array}{c}-0.000019 \\
(0.2511)\end{array}$ & $\begin{array}{l}0.0000134 \\
(0.1455)\end{array}$ & $\begin{array}{c}-0.001249 \\
(0.9002)\end{array}$ & $\begin{array}{c}-0.082191 \\
(0.4960)\end{array}$ & $\begin{array}{c}-0.264592 \\
(0.1574)\end{array}$ & \\
\hline ri, & 0.0000099 & $-0.1880 * *$ & -0.000001 & $-0.002 * * *$ & 0.0000065 & 0.008448 & 0.055565 & 0.258329 & \\
\hline & $(0.1827)$ & $(0.0106)$ & $(0.8644)$ & $(0.0041)$ & $(0.3561)$ & $(0.4352)$ & $(0.6204)$ & $(0.2080)$ & \\
\hline 2 & $0.000019 * * *$ & 0.018592 & 0.000008 & $-0.003 * * *$ & $0.00001 * *$ & $-0.0261 * *$ & 0.057648 & 0.17318 & \\
\hline & $(0.0089)$ & $(0.8156)$ & $(0.2943)$ & $(0.0051)$ & $(0.0385)$ & $(0.0486)$ & $(0.6498)$ & $(0.4470)$ & \\
\hline 3 & 0.0000129 & 0.001507 & -0.000003 & $-0.0002 * *$ & 0.0000107 & $-0.060 * * *$ & 0.016395 & -0.140498 & \\
\hline & $(0.0816)$ & $(0.9867)$ & $(0.6874)$ & $(0.0381)$ & $(0.1587)$ & $(0.0000)$ & $(0.8849)$ & $(0.5273)$ & \\
\hline 4 & $0.000018^{* *}$ & $-0.1463 * *$ & 0.000007 & $-0.0002 * *$ & 0.0000128 & $0.0216 * *$ & -0.074999 & -0.003946 & \\
\hline & $(0.0311)$ & $(0.0231)$ & $(0.3695)$ & $(0.0431)$ & $(0.1368)$ & $(0.0399)$ & $(0.5073)$ & $(0.9847)$ & \\
\hline 5 & $0.000024 * * *$ & -0.053046 & 0.0000115 & $-0.0002 * *$ & $0.00002^{* *}$ & 0.012642 & -0.040624 & -0.061609 & \\
\hline & $(0.0067)$ & $(0.4427)$ & $(0.1401)$ & $(0.012)$ & $(0.0114)$ & $(0.2467)$ & $(0.7256)$ & $(0.7867)$ & \\
\hline $\begin{array}{l}\text { Dummy1 - global } \\
\text { financial crisis (2007- } \\
\text { 2008) }\end{array}$ & $\begin{array}{l}0.000468 \\
(0.4560) \\
\end{array}$ & $\begin{array}{l}0.000566 \\
(0.9870) \\
\end{array}$ & $\begin{array}{c}0.0041 * * * \\
(0.0001)\end{array}$ & $\begin{array}{l}0.000436 \\
(0.4550)\end{array}$ & $\begin{array}{l}0.000546 \\
(0.6543) \\
\end{array}$ & $\begin{array}{l}0.000786 \\
(0.5543) \\
\end{array}$ & $\begin{array}{c}0.000496 \\
(0.5665) \\
\end{array}$ & $\begin{array}{l}0.000396 \\
(0.5550) \\
\end{array}$ & \\
\hline $\begin{array}{l}\text { Dummy2 - falling oil } \\
\text { prices (2014-2015) }\end{array}$ & $\begin{array}{l}0.000296 \\
(0.1232) \\
\end{array}$ & $\begin{array}{l}0.000776 \\
(0.2322)\end{array}$ & $\begin{array}{c}0.0017 * * * \\
(0.0000)\end{array}$ & $\begin{array}{l}0.0003 * * * \\
(0.0087) \\
\end{array}$ & $\begin{array}{l}0.000396 \\
(0.2672) \\
\end{array}$ & $\begin{array}{c}0.0023 * * * \\
(0.0000)\end{array}$ & $\begin{array}{c}-0.0036^{* *} \\
(0.0433)\end{array}$ & $\begin{array}{c}0.001296 \\
(0.619)\end{array}$ & \\
\hline
\end{tabular}

$(* * *)$ and $(* *)$ attached to coefficients denote the rejection of null hypotheses at the $1 \%$ and $5 \%$ statistical significance levels. 
The multivariate regression model output shows that systematic risk is significant in the majority of the series. This suggests the relevance of the Capital Asset Pricing Model (CAPM) in the valuation of oil and gas companies. In contrast to some other sectors of the economy, the book to market ratio and momentum factors do not seem to be relevant in the pricing of oil and gas stocks. HML factor was found significant at $1 \%$ level in seven stocks and at $5 \%$ level in three stocks. The momentum factor was not statistically significant in most of the models. The oil price risk is also significant in the determination of oil companies' stock returns at $1 \%$ significance level in the majority of the series. Similarly, there is an evidence that the coefficients of oil price increases are more significant in the series than that of oil price decreases (although both the oil price increases and decreases affect were found to affect stock returns as in Phan et al (2015b)) which is an indication of asymmetric effect. However, the significance was mainly observed in lags zero of both the oil price increases and decreases.

Surprisingly, the structural break created in the models to represent the global financial crisis between 2007 and 2008 had not been significant in the determination of oil companies' stock returns. The coefficients in the recent falling oil prices between 2014 and 2015 are found to be more significant in the models. Although, the effect of global financial crisis was seen to be serious as publicised, the impact of the recent falling oil prices has more effect on the oil companies' stock returns.

\subsection{Equality Test Between Brent Crude Price Increases and Decreases}

The impact of oil price increases and decreases on the stock returns of oil companies was tested using conventional F-tests. The following two null hypotheses were formulated and tested. 
Hypothesis 1 - There is no effects of Brent crude oil price increases on the oil companies' stock returns $\left(\beta_{\text {ioil }_{0}}{ }^{+}={\text {ioil }_{1}}^{+}={\beta_{\text {ioil }}}{ }^{+} \ldots \ldots \ldots \beta_{\text {ioil }_{5}}{ }^{+}=0\right)$.

Hypothesis 2 - There is no effects of Brent crude oil price decreases on the oil companies' stock returns $\left(\beta_{\text {ioil }_{0}}{ }^{-}={\text {ioil }_{1}}^{-}=\beta_{\text {ioil }_{2}}{ }^{-} \ldots \ldots \ldots{\text { ioil }_{5}}^{-}=0\right)$.

Results of the F-test to test the hypotheses are presented in Table 3.

Table 3 F-Tests Results of the Equality Hypotheses

\begin{tabular}{|c|c|c|c|c|c|}
\hline & $\begin{array}{l}\text { No effects of } \\
\text { Brent crude oil } \\
\text { price increases } \\
\text { on stock returns }\end{array}$ & $\begin{array}{l}\text { No effects of } \\
\text { Brent crude oil } \\
\text { price decreases } \\
\text { on stock returns }\end{array}$ & & $\begin{array}{l}\text { No effects of } \\
\text { Brent crude oil } \\
\text { price increases } \\
\text { on stock returns }\end{array}$ & $\begin{array}{l}\text { No effects of } \\
\text { Brent crude oil } \\
\text { price decreases } \\
\text { on stock returns }\end{array}$ \\
\hline Amec & 0.2476 & 0.1443 & Hardy Oil and Gas & 0.1461 & 0.1443 \\
\hline Aminex & $0.0462 * *$ & $0.0068 * * *$ & Royal D. Shell 'A' & 0.0725 & 0.4582 \\
\hline BG Group & $0.0000 * * *$ & $0.0000 * * *$ & Petrofac & 0.2409 & $0.0046 * * *$ \\
\hline $\mathrm{BP}$ & $0.0003 * * *$ & $0.0000 * * *$ & Lamprell & $0.0115 * *$ & $0.0000 * * *$ \\
\hline Cairn Energy & $0.0000 * * *$ & $0.0418 * *$ & Salamander Ener. & 0.8830 & 0.1031 \\
\hline Dragon Oil & $0.0095 * * *$ & $0.0022 * * *$ & Endeavor Intl. Co. & 0.9982 & 0.9708 \\
\hline Fortune Oil & 0.1376 & $0.0002 * * *$ & Kentz & 0.5803 & $0.0001 * * *$ \\
\hline Hunting & $0.0000 * * *$ & $0.0002 * * *$ & Heritage Oil & $0.0000 * * *$ & $0.0000 * * *$ \\
\hline JKX Oil and Gas & $0.0004 * * *$ & $0.0025 * * *$ & Cadogan Petr. & 0.2949 & $0.0000 * * *$ \\
\hline Premier Oil & $0.0000 * * *$ & $0.0000 * * *$ & Exillon Energy & $0.0000 * * *$ & $0.0185 * *$ \\
\hline Royal D. Shell 'B' & $0.0052 * * *$ & 0.4582 & Enquest & $0.0000 * * *$ & $0.0000 * * *$ \\
\hline Soco Intern. & $0.0000 * * *$ & $0.0050 * * *$ & Essar Energy & $0.0000 * * *$ & $0.0000 * * *$ \\
\hline Tullow Oil & $0.0000 * * *$ & $0.0000 * * *$ & Genel Energy & $0.0000 * * *$ & $0.0000 * * *$ \\
\hline Wood Gr. (John) & $0.0028 * * *$ & $0.0000 * * *$ & Ophir Energy & 0.1691 & 0.9647 \\
\hline Afren & $0.0000 * * *$ & $0.0000 * * *$ & Ruspetro & 0.7604 & 0.6759 \\
\hline
\end{tabular}

$(* * *)$ and $(* *)$ attached to $p$-values denote the rejection of null hypotheses at the $1 \%$ and $5 \%$ statistical significance levels.

The $p$-values of the F-test are mostly significant at $1 \%$ significance level to strongly reject the null hypotheses that oil prices increases or decreases have no effect on stock returns. In Royal Dutch Shell 'B' series, only oil price increases were found to have effect on stock returns. Conversely, the stock returns of Fortune oil, Kentz and Cadogan Petroleum were only affected by decreases in oil price. These few companies show a slight evidence that oil price exposure depends partly on the risk characteristics of individual companies. Although, this cannot be confirmed on the majority of the series under study. 


\section{Findings}

Firstly, our findings suggest that oil price shock has an effect on the oil and gas companies' stock returns. We found an evidence for the existence of asymmetric effect in the Brent crude oil price with oil price increases being more significant than oil price decreases. Secondly, the market factor is significant in almost all the oil and gas stocks. The finding demonstrates the importance of systematic risk in the determination of the excess return of the individual stocks. Thirdly, the construction of portfolios by buying stocks of small firms and short selling stocks of large firms could be a beneficial investment strategy in the London stock exchange. Fourthly, there is no clear evidence that using book to market ratio can be a successful strategy in the oil and gas sector. Fifthly, the formation of portfolios based on momentum will not be a useful strategy in the oil and gas sector. Lastly, the global financial crisis of 2007 was not found to have a significant impact in the determination of oil companies' stock returns compared to the recent falling oil prices in 2014 through to the end of 2015. Our findings are similar to those of Moya-Martinez et al (2014), Faff and Brailsford (1999), Mohanty and Nandha (2011) and Broadstock et al (2016).

\section{Conclusion}

The shock of crude oil price and its impact on stock returns in the oil and gas sector have been researched extensively due to its importance to the overall economy. This paper aimed to analyse the determinants of stock returns in the UK oil and gas sector and to explore the possibility of using fundamentals and company specific information in asset pricing. The oil price shock was decomposed into five lags each of oil price increases and decreases to assess whether there is the existence of asymmetric effect. We also used systematic risk (beta), firm's size, 
book-to-market ratio, momentum and the decomposed oil price shocks as explanatory variables in the asset pricing model to investigate their significance in the determination of oil companies' stock returns. The results from this analysis suggest oil price changes, market risk and firm's size explain the variation of stock returns in the oil and gas sector. The book to market ratio and momentum effect were not found to be significantly affecting the stock returns in the oil and gas sector. We believe this information can be used by investors while choosing stocks from the oil and gas sector for their investment portfolios.

\section{References}

Arouri, M. 2011. Does crude oil move stock markets in Europe? A sector investigation. Economic Modeling 28: 1716-1725.

Asteriou, D. \& Bashmakova, Y. 2013. Assessing the impact of oil returns on emerging stock markets: A panel data approach for ten Central and Eastern European Countries. Energy Economics, vol. 38, pp. 204-211.

Broadstock, D. C., Fan, Y., Ji, Q. and Zhang, D. 2016. Shocks and stocks: A bottomup assessment of the relationship between oil prices, gasoline prices and the returns of Chines firms. The Energy Journal 37: 1-32.

Carhart, Mark M. 1997. On persistence in mutual fund performance. The Journal of Finance 52 (1): 57-82.

Chou, P., Ho, P. \& Ko, K. 2012. Do industries matter in explaining stock returns and asset-pricing anomalies? Journal of Banking \& Finance, vol. 36, no. 2, pp. 355370.

Elyasiani, Elyas, Iqbal Mansur, and Babatunde Odusami. 2011. Oil price shocks and industry stock returns. Energy Economics 33 (5) (9): 966-74.

Faff, Robert W., and Timothy J. Brailsford. 1999. Oil price risk and the australian stock market. Journal of Energy Finance \& Development 4 (1) (6): 69-87.

Fama, Eugene F., and Kenneth R. French. 1993. Common risk factors in the returns on stocks and bonds. Journal of Financial Economics 33 (1) (2): 3-56.

Filis, G., Degiannakis, S. \& Floros, C. 2011. Dynamic correlation between stock market and oil prices: The case of oil-importing and oil-exporting countries. International Review of Financial Analysis, vol. 20, no. 3, pp. 152-164.

Gregory, A., Tharayan, R. and Christidis, A. 2013. Constructing and testing alternative versions of the Fama-French and carhart models in the UK. Journal of Business Finance \& Accounting 40 (1-2): 172-214. 
Lawrence, Edward R., John Geppert, and Arun J. Prakash. 2007. Asset pricing models: A comparison. Applied Financial Economics 17 (11) (07): 933-40.

Lintner, John. 1965. Security prices, risk, and maximal gains from diversification. The Journal of Finance 20 (4): 587-615.

Manning, DN. 1991. Petrol prices, oil price rises and oil price falls: Some evidence for the UK since 1972. Applied Economics 23 (9): 1535-41.

Markowitz, Harry. 1952. Portfolio selection*. The Journal of Finance 7 (1): 77-91.

Melichar, M. 2016. Energy price shocks and economic activity: Which energy price series should we be using? Energy Economics 54: 431-443.

Mendoza, O. and Vera, D. 2010. The asymmetric effects of oil shocks on an oilexporting economy. Cuadernos De Economia 47: 3-13.

Mohanty, Sunil K., and Mohan Nandha. 2011. Oil risk exposure: The case of the US oil and gas sector. Financial Review 46 (1): 165-91.

Mohanty, Sunil, Mohan Nandha, and Gabor Bota. 2010. Oil shocks and stock returns: The case of the Central and Eastern European (CEE) oil and gas sectors. Emerging Markets Review 11 (4) (12): 358-72.

Mohanty, Sunil, Mohan Nandha, Essam Habis, and Eid Juhabi. 2014. Oil price risk exposure: The case of the U.S. travel and leisure industry. Energy Economics 41 (0) (1): 117-24.

Mork, K. A., Olsen, O. and Mysen, H. T. 1994. Macroeconomic responses to oil price increases and decreases in seven OECD countries. The Energy Journal 15 (4): 1935.

Mossin, Jan. 1966. Equilibrium in a capital asset market. Econometrica: Journal of the Econometric Society: 768-83.

Narayan, P. K. and Sharma, S. S. 2011. New evidence on oil price and firm returns. Journal of Banking and Finance 35: 3253-3262.

Phan, D. H. B., Sharma, S. S. and Narayan, P. K. 2015(a). Stock return forecasting: Some new evidence. International Review of Financial Analysis 40: 38-51

Phan, D. H. B., Sharma, S. S. and Narayan, P. K. 2015(b). Oil price and stock returns of consumers and producers of crude oil. Journal of International Financial Markets, Institutions and Money 34: 245-262.

Ramos, S. B., \& Veiga, H. 2011. Risk factors in oil and gas industry returns: International evidence. Energy Economics, 33(3), 525-542.

Sharpe, William F. 1964. Capital asset prices: A theory of market equilibrium under conditions of risk*. The Journal of Finance 19 (3): 425-42.

Wang, Y., Wu, C. \& Yang, L. 2013. Oil price shocks and stock market activities: Evidence from oil-importing and oil-exporting countries. Journal of Comparative Economics, vol. 41, no. 4, pp. 1220-1239. 